\title{
Homotopy perturbation method for biological species living together
}

\author{
J. Biazar *, Z. Ayati, M. Partovi \\ Department of Applied Mathematics, Faculty of Mathematical Science, University of Guilan, \\ P.O. Box 41635-19141, P.C. 41938336997, Rasht, Iran \\ *Corresponding author E-mail: : Biazar@guilan.ac.ir
}

\begin{abstract}
In this paper, Homotopy perturbation method has been used for solving systems of nonlinear integro-differential equations governing on the problem of results reveal that the method is very effective and simple. The results of homotopy perturbation method are of high accuracy. The example is presented to show the ability of the method.
\end{abstract}

Keywords: Homotopy perturbation method, Integro-differential equations, integro-differential equations, Biological species.

\section{Introduction}

Consider two separate species with numbers $X(t)$ and $Y(t)$ at time $t$ where first species increases and the second decrease. If they are put together, assuming that the second species will feed on the first, there will be an increase in the rate of the second species $d Y / d t$ which depends not only on the present population $X(t)$, but also on all previous values of the first species. When a steady-state condition is reached between these two species, it is described by the following system of two integro-differential equations:

$$
\left\{\begin{array}{lll}
\frac{d X(t)}{d t}=X(t)\left[k_{1}-\gamma_{1} Y(t)-\int_{t-T_{0}}^{t} f_{1}(t-s) Y(s) d s\right] & k_{1}>0, \\
\frac{d Y(t)}{d t}=Y(t)\left[-k_{2}+\gamma_{2} X(t)+\int_{t-T_{0}}^{t} f_{2}(t-s) X(s) d s\right] & k_{2}>0 .
\end{array}\right.
$$

Where $k_{1}$ and $-k_{2}$ are the coefficients of increaseing and decreaseing of the first and the second species (had they staged separate), respectively. The parameters $\gamma_{1}, f_{1}$, and $\gamma_{2}, f_{2}$, depend on the respective species. The main problem of biological species living together is presented in [1-2].

Recently, a great deal of interest has been focused on the applications of the homotopy perturbation method, well addressed in [3-9]. This method has been used to solve wide variety mathematical problems under study. Almost all perturbation methods are based on the assumption of the existence of a small parameter in the equation. But most nonlinear problems have not such a small parameter. This method has been proposed to eliminate the small parameter [10-11]. Also, this method has been used to solve hyperbolic differential equations [12], and to other equations [13-14]. In this article, we use Homotopy perturbation method to solve systems of integro-differential equations.

\section{Basic idea of homotopy perturbation method}

To illustrate the basic ideas of homotopy perturbation method for solving nonlinear differential equations, we consider the following nonlinear functional equation: 


$$
A(u)-f(r)=0, \quad r \in \Omega,
$$

with the following boundary conditions:

$$
B\left(u, \frac{\partial u}{\partial n}\right)=0, \quad r \in \Gamma,
$$

where $\mathrm{A}$ is a general functional operator, $B$ is a boundary operator, $f(r)$ is a known analytical function, and $\Gamma$ is the boundary of the domain $\Omega$, and $\partial / \partial n$ denotes differentiation along the normal drawn outwards from $\Omega$. Generally speaking the operator A can be divided into two parts $L$ and $N$, where $L$ is a linear and $N$ is a nonlinear operators. Eq. (2), therefore, can be rewritten as the following:

$$
L(u)+N(u)-f(r)=0 .
$$

We construct a homotopy for Eq. (2), $v(r, p): \Omega \times[0,1] \rightarrow \mathbb{R}$, which satisfies:

$$
H(v, p)=(1-p)\left[L(v)-L\left(u_{0}\right)\right]+p[A(v)-f(r)]=0, \quad p \in[0,1], \quad r \in \Omega,
$$

or

$$
H(v, p)=L(v)-L\left(u_{0}\right)+p L\left(u_{0}\right)+p[N(v)-f(r)]=0,
$$

Where $p \in[0,1]$ is an embedding parameter, $u_{0}$ is an initial approximation of Eq. (2), which satisfies the boundary conditions. Obviously, form Eqs. (5) and (6) we will have

$$
\begin{aligned}
& H(v, 0)=L(v)-L\left(u_{0}\right)=0, \\
& H(v, 1)=A(v)-f(r)=0 .
\end{aligned}
$$

Thus, the changing process of $p$ from zero to unity is just that of $v(r, p)$ from $u_{0}$ to $u_{r}$. In topology, these are called deformation, and $L(v)-L\left(u_{0}\right), A(v)-f(r)$ are called homotopic. Here the embedding parameter is introduced much more naturally, unaffected by artificial factors; further it can be considered as small parameter for $0 \leq p \leq 1$. So it is very natural to assumed that the solution of Eqs. (5) and (6) can be written as a power series in $p$ :

$$
v=v_{0}+p v_{1}+p^{2} v_{2}+\cdots
$$

Setting $p=1$, results in the approximate solution of Eq. (2)

$$
u=\lim _{p \rightarrow 1} v=v_{0}+v_{1}+v_{2}+\cdots
$$

\section{Homotopy perturbation method for system (1)}

To apply homotopy perturbation method for system (1), functions $f_{1}(t-s)$ and $f_{2}(t-s)$ should be determined. So let

$$
\begin{gathered}
f_{1}(t-s)=f_{2}(t-s)=e^{-(t-s)}, \\
X_{0}(t)=N_{1}, \quad Y_{0}(t)=N_{2} .
\end{gathered}
$$

Corresponding homotopies can be readily constructed as the following: 


$$
\left\{\begin{array}{l}
(1-p)\left(\frac{d X}{d t}-\frac{d u_{0}}{d t}\right)+p\left(\frac{d X}{d t}-X(t)\left[k_{1}-\gamma_{1} Y(t)-\int_{t-T_{0}}^{t} f_{1}(t-s) Y(s) d s\right]\right)=0 \\
(1-p)\left(\frac{d Y}{d t}-\frac{d v_{0}}{d t}\right)+p\left(\frac{d Y}{d t}-Y(t)\left[-k_{2}+\gamma_{2} X(t)+\int_{t-T_{0}}^{t} f_{2}(t-s) X(s) d s\right]\right)=0 .
\end{array}\right.
$$

Equating the coefficients of the terms with identical powers of $p$, we lead to;

$$
\begin{aligned}
& p^{0}:\left\{\begin{array}{l}
X_{0}=N_{1}, \\
Y_{0}=N_{2},
\end{array}\right. \\
& p^{1}:\left\{\begin{array}{l}
X_{1}(t)=\left[N_{1} k_{1}-\gamma_{1} N_{1} N_{2}-N_{1} N_{2}\left(1-e^{-T_{0}}\right)\right] t \\
Y_{1}(t)=\left[-N_{2} k_{2}+\gamma_{2} N_{1} N_{2}+N_{1} N_{2}\left(1-e^{-T_{0}}\right)\right] t
\end{array}\right. \\
& P^{2}:\left\{\begin{array}{l}
X_{2}(t)=k_{1}\left(N_{1} k_{1}-\gamma_{1} N_{1} N_{2}-N_{1} N_{2}\left(1-e^{-T_{0}}\right)\right)+N_{1} e^{-T_{0}}\left(\gamma_{2} N_{1} N_{2}\right. \\
\left.-N_{2} k_{2}+N_{1} N_{2}\left(1-e^{-T_{0}}\right)\right)+N_{2} e^{-T_{0}}\left(N_{1} k_{1}-\gamma_{1} N_{1} N_{2}-N_{1} N_{2}\left(1-e^{-T_{0}}\right)\right) \\
-\gamma_{1} N_{2}\left(N_{1} k_{1}-\gamma_{1} N_{1} N_{2}-N_{1} N_{2}\left(1-e^{-T_{0}}\right)\right)-\gamma_{1} N_{1}\left(\gamma_{2} N_{1} N_{2}-N_{2} k_{2}+\right. \\
\left.\left.N_{1} N_{2}\left(1-e^{-T_{0}}\right)\right)-N_{1}\left(\gamma_{2} N_{1} N_{2}-N_{2} k_{2}+N_{1} N_{2}\left(1-e^{-T_{0}}\right)\right)-N_{2}\left(N_{1} k_{1}\right)\right), \\
Y_{2}(t)=k_{2} N_{2}\left(k_{2}-\gamma_{2} N_{1}-N_{1}\left(1-e^{-T_{0}}\right)\right)+N_{1} N_{2} \gamma_{2}\left(k_{1}-N_{2} \gamma_{1}-\right. \\
\left.N_{2}\left(1-e^{-T_{0}}\right)\right)-\gamma_{2} N_{1} N_{2}\left(k_{2}-\gamma_{2} N_{1}-N_{1}\left(1-e^{-T_{0}}\right)\right)+N_{1} N_{2} e^{-T_{0}}\left(k_{2}-\right. \\
\left.\gamma_{2} N_{1}-N_{1}\left(1-e^{-T_{0}}\right)\right)-N_{1} N_{2} e^{-T_{0}}\left(k_{1}-N_{2} \gamma_{1}-N_{2}\left(1-e^{-T_{0}}\right)\right)-N_{1} N_{2} \\
\left(k_{2}-\gamma_{2} N_{1}-N_{1}\left(1-e^{-T_{0}}\right)\right)+N_{1} N_{2}\left(k_{1}-N_{2} \gamma_{1}-N_{2}\left(1-e^{-T_{0}}\right)\right),
\end{array}\right.
\end{aligned}
$$

So the exact solutions of the system (1) can be entirely determined. However, in practice, all terms of the series cannot be determined; in this study we use three-terms approximations:

$$
\begin{aligned}
& X(t)=\sum_{i=0}^{\infty} p^{i} X_{i}, \\
& Y(t)=\sum_{i=0}^{\infty} p^{i} Y_{i} .
\end{aligned}
$$

As $p \rightarrow 1$, (12) tends to system (1) and (14) and (15), in most cases converges to an approximate solution of system (1), i.e.

$$
\begin{aligned}
& X=\lim _{p \rightarrow 1} X=X_{0}+X_{1}+\cdots, \\
& Y=\lim _{p \rightarrow 1} Y=Y_{0}+Y_{1}+\cdots
\end{aligned}
$$

Three-terms approximations for $X$ and $Y$ are as follows: 


$$
\begin{aligned}
& X(t)=N_{1}+N_{1}\left[k_{1}-\gamma_{1} N_{2}-N_{2}\left(1-e^{-T_{0}}\right)\right] t+0.5\left[k _ { 1 } \left(N_{1} k_{1}-\gamma_{1} N_{1} N_{2}\right.\right. \\
& \left.-N_{1} N_{2}\left(1-e^{-T_{0}}\right)\right)+N_{1} e^{-T_{0}}\left(\gamma_{2} N_{1} N_{2}-N_{2} k_{2}+N_{1} N_{2}\left(1-e^{-T_{0}}\right)\right)+N_{2} e^{-T_{0}} \\
& \left(N_{1} k_{1}-\gamma_{1} N_{1} N_{2}-N_{1} N_{2}\left(1-e^{-T_{0}}\right)\right)-\gamma_{1} N_{2}\left(N_{1} k_{1}-\gamma_{1} N_{1} N_{2}-N_{1} N_{2}\right. \\
& \left.\left(1-e^{-T_{0}}\right)\right)-\gamma_{1} N_{1}\left(\gamma_{2} N_{1} N_{2}-N_{2} k_{2}+N_{1} N_{2}\left(1-e^{-T_{0}}\right)\right)-N_{1}\left(\gamma_{2} N_{1} N_{2}-\right. \\
& \left.\left.N_{2} k_{2}+N_{1} N_{2}\left(1-e^{-T_{0}}\right)\right)-N_{2}\left(N_{1} k_{1}-\gamma_{1} N_{1} N_{2}-N_{1} N_{2}\left(1-e^{-T_{0}}\right)\right)\right] t^{2} \\
& Y(t)=N_{2}-N_{2}\left[k_{2}-\gamma_{2} N_{1}-N_{1}\left(1-e^{-T_{0}}\right)\right] t+0.5\left[k _ { 2 } N _ { 2 } \left(k_{2}-\gamma_{2} N_{1}-\right.\right. \\
& \left.N_{1}\left(1-e^{-T_{0}}\right)\right)+N_{1} N_{2} \gamma_{2}\left(k_{1}-N_{2} \gamma_{1}-N_{2}\left(1-e^{-T_{0}}\right)\right)-\gamma_{2} N_{1} N_{2}\left(k_{2}-\right. \\
& \left.\gamma_{2} N_{1}-N_{1}\left(1-e^{-T_{0}}\right)\right)+N_{1} N_{2} e^{-T_{0}}\left(k_{2}-\gamma_{2} N_{1}-N_{1}\left(1-e^{-T_{0}}\right)\right)-N_{1} N_{2} e^{-T_{0}} \\
& \left(k_{1}-N_{2} \gamma_{1}-N_{2}\left(1-e^{-T_{0}}\right)\right)-N_{1} N_{2}\left(k_{2}-\gamma_{2} N_{1}-N_{1}\left(1-e^{-T_{0}}\right)\right)+ \\
& \left.N_{1} N_{2}\left(k_{1}-N_{2} \gamma_{1}-N_{2}\left(1-e^{-T_{0}}\right)\right)\right] t^{2} .
\end{aligned}
$$

\section{Numerical results}

If we know the values of parameters $k_{1}, k_{2}, N_{1}, N_{2}, \gamma_{1}$, and $\gamma_{2}$, we can derive the values of $X$ and $Y$. For numerical results we use the following values and get $X$ and $Y$ in terms of $t$. That the following values for the parameters have been closen. There is no any advantage for these values

$$
k_{1}=0.02, k_{2}=\gamma_{1}=\gamma_{2}=0.01, N_{1}=N_{2}=10, T_{0}=0.1
$$

So three-terms approximations for $X$ and $Y$ are as follows:

$$
\left\{\begin{array}{l}
X(t)=10-10.6162 t-0.1557 t^{2} \\
Y(t)=10+10.4163 t+5 \times 10^{-4} t^{2}
\end{array}\right.
$$

Some values of $Z(t)$ and $Y(t)$ are listed in the following table 1, For comparison with the results done by Biazar [2].

Table 1: Numerical results of equations (11) for system (1)

\begin{tabular}{rrrrrrrrrr}
\hline$t$ & 0.01 & 0.03 & 0.05 & 0.07 & 0.1 & 0.3 & 0.5 & 0.7 & 0.9 \\
\hline$X$ & 9.8968 & 9.6904 & 9.4838 & 9.2771 & 8.9668 & 6.8911 & 4.8030 & 2.7023 & 0.5892 \\
$Y$ & 10.1042 & 10.3125 & 10.5208 & 10.7291 & 11.0416 & 13.1250 & 15.2082 & 17.2916 & 19.3750 \\
\hline
\end{tabular}

\section{Conclusion}

In this article, we have applied homotopy perturbation method for solving the nonlinear systems integro-differential equations. In many cases one or two terms approximations have enough accuracy, i.e. fast convergence which can be mentioned as an advantage of the method. Homotopy perturbation method has been known as a powerful device for solving many functional equations such as ordinary, partial differential equations, integral equations and so many other equations. There is less computations needed in comparison with the Adomian decomposition method. In our work, for computations we used Maple 13. 


\section{References}

[1] J. Abdul, Introduction to Integral Equations with Applications, Wiley, New York, 1999.

[2] E. Babolian, J. Biazar, Solving the problem of biological species living together by Adomian Decomposition method, Applied Mathematics and Computation 129 (2002) 339-343.

[3] J. Biazar, H. Ghazvini, M.Eslami, He's Homotopy perturbation method for systems of integro- differential equations, Chaos, solitons and Fractals 39 (2009) 1253-1258.

[4] J. Biazar, H. Ghazvini Exact solutions for nonlinear Schrödinger equations by He's homotopy perturbation method, Physics Letters A 366 (2007) 79-84.

[5] J. H. He, The homotopy perturbation method for nonlinear oscillators with discontinuities, Applied Mathematics and Computation 151 (2004) 287-292.

[6] S. Abbasbandy, Application of He's homotopy perturbation method to functional integral equations, Chaos, solitons and Fractals 31(5) (2007) 1243-1247.

[7] J. H. He, Asymptology by homotopy perturbation method, Applied Mathematics and Computation 156 (3) (2004) 591-596.

[8] J. H. He, Homotopy Perturbation method for bifurcation of nonlinear problems, International Journal of Nonlinear Science Numerical Simulation 6 (2) (2005) 207-208.

[9] J. H. He, New interpretation of homotopy perturbation method, Internatinal Journal of Modern physics B 20 (2006) 1141-99.

[10] SJ. Liao, An approximate solution technique not depending on small parameter: a special example, International Journal of Non-Linear Mechanics, 30(3) (1995) 371-80.

[11] SJ. Liao, Boundary element method for general nonlinear differential operators, Engineering Analysis with Boundary Elements 20(2) (1997) 91-9.

[12] J. Biazar, H. Ghazvini He's homotopy perturbation method for solving hyperbolic partial differential equations, Computers and Mathematics with Applications 56 (2008) 453-458.

[13] J. Biazar, H. Ghazvini, Numerical solution for special Nonlinear Fredholm integral equation by He's homotopy perturbation method. Applied Mathematics and Computation 195 (2008) 681-687.

[14] J. Biazar, H. Ghazvini, He's homotopy perturbation method for solving system of Volterra integral equations of the second kind, Chaos, Solitons and Fractals 39(2) (2009) 770-777. 